\title{
The Flow Regimes of the Fuel Water Coal Slurries in the Canals of Spraying Devices
}

\author{
Ahmet K. Dzhundubaeva, \\ Myrzatay S. Sultanaliyev ${ }^{\text {a }}$, Vasily I. Murko, \\ Ludmila V. Kulagina ${ }^{\mathfrak{c}}$ and Marina P. Baranova ${ }^{\mathrm{d}}$ \\ ${ }^{a}$ Research Institute of Energy and Economics \\ under the State Committee for Energy of the Kyrgyz Republic \\ 119 Ahunbaeva, Bishkek, 720055, Kyrgyz Republic \\ ${ }^{b}$ Sibecotechnika \\ 2 Kommunarov, Novokuznetsk, 654079, Russia \\ 'Siberian Federal University \\ 79 Svobodny, Krasnoyarsk, 660041, Russia \\ ${ }^{d}$ Krasnoyarsk State Agrarian University \\ 90 Mira, Krasnoyarsk, 660049, Russia
}

Received 26.06.2017, received in revised form 21.09.2017, accepted 12.01.2018

The article deals with the flow of highly concentrated water-coal fuel slurries (WCS) when they are added for the combustion through spraying devices - nozzles. The flow regimes of slurries in thin canals of spraying devices are studied. It is established that during the WCS flow through thin canals, the flow regime is violated, which is caused by the mutual influence of geometric dimensions and flow parameters of the slurries when it is burned in combustion chambers of power plants. Therefore, the design dimensions, especially the outlet diameters of the nozzle holes, must be selected, taking into account the obtained analytical expressions, which should be refined in carrying out model and full-scale experiments with specific types of WCS of constant composition.

Keywords: Water coal slurry, thin canals of spraying devices, flow regimes.

Citation: Dzhundubaev A.K., Sultanaliyev M.S., Murko V.I., Kulagin V.A., Baranova M.P. The flow regimes of the fuel water coal slurries in the canals of spraying devices, J. Sib. Fed. Univ. Eng. technol., 2018, 11(2), 242-249. DOI: 10.17516/1999-494X0027.

(c) Siberian Federal University. All rights reserved

* Corresponding author E-mail address: v.a.kulagin@mail.ru 


\title{
Режимы течения топливных водоугольных суспензий в каналах распыливающих устройств
}

\author{
А.К. Джундубаев ${ }^{\text {a }}$ М.С. Султаналиев ${ }^{a}$, \\ В.И. Мурко ${ }^{\sigma}$, Л.В. Кулагина ${ }^{\text {, М.П. Баранова }}$ \\ ${ }^{a}$ НИИ энергетики и экономики \\ при Госкомпромэнергонедр Киргизской Республики \\ Кыргызская Республика, 720055, Бишкек, Ахунбаева, 119 \\ бЗАО «Сибэкотехника» \\ Россия, 654079, Новокузнецк, Коммунаров, 2 \\ ${ }^{8}$ Сибирский федеральныий университет \\ Россия, 660041, Красноярск, пр. Свободный, 79 \\ ${ }^{2}$ Красноярский государственный аграрный университет \\ Россия, 660049, Красноярск, пр. Мира, 90
}

B cтатье рассмотрены вопросы течения высококонцентрированных водоугольных топливных суспензий при подаче их на сжигание через распыливаюшче устройства форсунки. Исследованы режимы течения суспензий в тонких каналах распьливающих устройств. Установлено, что при течении ВУС по тонким каналам нарушается режим течения, что обусловлено взаимным влиянием геометрических размеров и параметров течения суспензии при организации ее сжигания в топочных камерах энергоустановок. Поэтому конструктивные размеры, особенно выходные диаметры отверстий форсунок, необходимо подбирать, учитывая полученные аналитические выражения, которые должны быть уточнены при проведении модельных и натурных экспериментов с конкретными типами ВУС постоянного состава.

Ключевые слова: Водоугольные суспензии, тонкие каналь распьливающих устройств, режимы течения.

Применение водоугольных суспензий (ВУС) в качестве топлива для промышленных энергоустановок, особенно в последние годы, привлекает к ним интерес многочисленных исследователей. Большинство работ посвящено проблемам, связанным с гидродинамикой течения высококонцентрированных ВУС применительно к магистральным трубопроводным системам. Однако ряд технических трудностей, возникающих при организации внутрицехового распределения ВУС на тепловых электростанциях и, главное, при распыливании их через специальные форсунки в топке или аппарате предварительного отделения влаги обусловлен недостаточной изученностью параметров и режимов течения ВУС в этих условиях.

Цель работы - получение аналитических выражений, позволяющих определять геометрические размеры каналов сопел распыливающих форсунок ВУС с учетом параметров течения.

Для достижения поставленной цели проводили исследования режимов течения высококонцентрированных ВУС в тонких каналах распыливающих устройств.

Высококонцентрированная ВУС представляет собой сложную сплошную среду, свойства которой определяются такими параметрами, как влажность, зерновая характеристика исходной угольной пыли, присутствие поверхностно-активных добавок, физико-химические свойства 
угля и т.д. [1-4]. В работе [5] установлены причины стабилизации водоугольных суспензий. Как показывает опыт, ВУС обладает свойствами, присущими неньютоновской жидкости степенного вида, реологическое уравнение которой имеет вид

$$
\tau=k \gamma^{n}
$$

где $\tau$ - касательное напряжение; $\gamma$ - скорость деформации; $k$ и $n$ - постоянные коэффициенты, причем $n<1[6,7]$.

Зависимость (1) приводит к значительному усложнению уравнения движения ВУС по сравнению с ньютоновскими жидкостями. Однако в некоторых практических случаях движение ВУС может быть описано обычными уравнениями. При этом для конкретных технических задач необходимо учитывать реологические особенности ВУС. В качестве исходных примем уравнения динамики сплошной среды «в напряжениях» и уравнение несжимаемости, которые справедливы для любой сплошной среды:

$$
\begin{aligned}
& \rho \frac{d U}{d t}=\rho F_{x}+\frac{\partial P_{x x}}{\partial x}+\frac{\partial P_{y x}}{\partial y}+\frac{\partial P_{z x}}{\partial z} \\
& \rho \frac{d V}{d t}=\rho F_{y}+\frac{\partial P_{x y}}{\partial x}+\frac{\partial P_{y y}}{\partial y}+\frac{\partial P_{z y}}{\partial z} \\
& \rho \frac{d W}{d t}=\rho F_{z}+\frac{\partial P_{x z}}{\partial x}+\frac{\partial P_{y z}}{\partial y}+\frac{\partial P_{z z}}{\partial z} \\
& \frac{\partial U}{\partial x}+\frac{\partial V}{\partial y}+\frac{\partial W}{\partial z}=0
\end{aligned}
$$

где $U, V, W$ - проекции скорости на оси координат; $P_{y x}, P_{z x}, P_{z y}$ - касательные напряжения; первый подстрочный индекс обозначает ось, перпендикулярно которой ориентирована площадка, второй индекс - ось, на которую спроектировано это напряжение.

$$
\begin{aligned}
& P_{x x}=\vec{P}_{x} \\
& P_{y y}=\vec{P}_{y} \quad \text { нормальные напряжения. } \\
& P_{z z}=\vec{P}_{z}
\end{aligned}
$$

Если вязкость $\mu=$ const, то полученные уравнения носят название уравнений Стокса. Их обычно записывают в компактной векторной форме:

$$
\left.\begin{array}{l}
\frac{\partial \vec{V}}{\partial t}+(\vec{V} \Delta)=\vec{F}-\frac{1}{\rho} \operatorname{grad} P+v \Delta^{2} \vec{V} \\
\operatorname{div} \vec{V}=0,
\end{array}\right\}
$$

где $v=\frac{\mu}{\rho}-$ кинематическая вязкость.

Рассмотрим случай слоистого течения, причем скорость в продольном направлении не изменяется, а зависимость носит линейный характер. Как показывают опыты, это обстоятельство справедливо в ограниченном диапазоне скоростей, характерных для движения ВУС по трубопроводам и через регулирующую арматуру. Наиболее общим видом связи между тензо- 
ром напряжений $\mathrm{P}$ и тензором скоростей деформации $\mathrm{S}$ как для изотропной, так и для анизотропной жидкости будет выражение

$$
P=a S+b \varepsilon
$$

где $a$ и $b$ - скаляры, имеющие смысл вязкости и давления соответственно [8].

Анизотропность среды будет учитываться $\mu$, который в общем случае также является тензором. Запись выражения (6) может быть использована в данной постановке задачи. Это приводит к тому, что

$$
\begin{aligned}
& P_{x x}=-P+2 \mu \frac{\partial U}{\partial x} ; \\
& P_{y y}=-P+2 \mu \frac{\partial V}{\partial y} ; \\
& P_{z z}=-P+2 \mu \frac{\partial W}{\partial z} ; \\
& P_{x x}=P_{y x}=\mu\left(\frac{\partial U}{\partial y}+\frac{\partial V}{\partial x}\right) ; \\
& P_{y z}=P_{z y}=\mu\left(\frac{\partial V}{\partial z}+\frac{\partial W}{\partial y}\right) ; \\
& P_{x x}=P_{y x}=\mu\left(\frac{\partial W}{\partial x}+\frac{\partial U}{\partial z}\right) .
\end{aligned}
$$

Вопрос о вязкости ВУС остается наиболее сложным. Теоретические представления, приведенные в работах $[6,9]$, показывают, что для жидкости, обладающей структурой, вязкость определяется из выражения

$$
\mu(\tau)=\mu_{m}+\alpha\left(\mu_{o}-\mu_{m}\right)
$$

где $\mu_{m}$ - наименьшая вязкость предельно разрушенной структуры; $\mu_{o}$ - наибольшая вязкость практически неразрушенной структуры; $\alpha$ - степень тиксотропного восстановления структуры в точке, зависящей от скорости деформации.

Чаще всего эта зависимость представляется линейной:

или

$$
\mu=\mu_{o}-k \frac{d U}{d y}
$$

$$
\mu=\mu_{o}+\frac{1}{k \frac{d U}{d y}},
$$

где $k$ - коэффициент, который зависит от структуры ВУС и внешнего воздействия - напряжения сдвига.

Последнее равенство справедливо для достаточно узкого диапазона скоростей. Для гидродинамики течения ВУС главную роль играет второе слагаемое выражений (10) и (11). Интересно отметить, что для высококонцентрированных дисперсных систем известны случаи возрастания вязкости с увеличением скорости деформации [10]. Такое различие 
результатов зависит не только от содержания твердой фазы, но и от режима течения ВУС [4].

Представление $\mu$ может быть обобщено на трехмерное пространство. Однако в конечном счете можно ограничиться слоистым одномерным течением. Подставляя значения напряжений в уравнение (2), получим

$$
\begin{aligned}
& \frac{\partial P_{x x}}{\partial x}+\frac{\partial P_{y x}}{\partial y}+\frac{\partial P_{z x}}{\partial x}=-2 \mu \frac{\partial^{2} U}{\partial x^{2}}+2 \frac{\partial \mu \partial^{2} U}{\partial x \partial x}+\mu\left(\frac{\partial^{2} U}{\partial y^{2}}+2 \frac{\partial^{2} V}{\partial x \partial y}\right)+ \\
& +\frac{\partial \mu}{\partial y}\left(\frac{\partial U}{\partial y}+\frac{\partial V}{\partial x}\right)+\mu\left(\frac{\partial^{2} W}{\partial x \partial z}+\frac{\partial^{2} U}{\partial z^{2}}\right)+\frac{\partial \mu}{\partial z}\left(\frac{\partial W}{\partial x}+\frac{\partial U}{\partial z}\right)= \\
& =-\frac{\partial P}{\partial x}+\mu\left(\frac{\partial^{2} U}{\partial x^{2}}+\frac{\partial^{2} U}{\partial y^{2}}+\frac{\partial^{2} U}{\partial z^{2}}\right)+\mu \frac{\partial}{\partial x}\left(\frac{\partial U}{\partial x}+\frac{\partial V}{\partial y}+\frac{\partial W}{\partial z}\right)+ \\
& 2 \frac{\partial \mu \partial U}{\partial x \partial x}+\frac{\partial \mu}{\partial y}\left(\frac{\partial U}{\partial y}+\frac{\partial V}{\partial x}\right)+\frac{\partial \mu}{\partial z}\left(\frac{\partial W}{\partial x}+\frac{\partial U}{\partial z}\right)=\frac{\partial P}{\partial x}+\mu \Delta^{2} U
\end{aligned}
$$

так как

$$
\frac{\partial U}{\partial x}+\frac{\partial V}{\partial y}+\frac{\partial W}{\partial z}=\operatorname{div} \vec{V}=0
$$

(в силу уравнения неразрывности),

$$
2 \frac{\partial \mu}{\partial x} \frac{\partial U}{\partial x}=\frac{\partial \mu}{\partial y}\left(\frac{\partial U}{\partial y}+\frac{\partial V}{\partial x}\right)=\frac{\partial \mu}{\partial z}\left(\frac{\partial W}{\partial x}+\frac{\partial U}{\partial z}\right)=0
$$

(в силу принятого предположения о линейности $\mu$ ).

Аналогичные преобразования можно провести для остальных проекций. В результате вид полученных выражений совпадает с уравнениями Стокса

$$
\begin{aligned}
& \frac{\partial \vec{V}}{\partial t}+(\vec{V}) \vec{V}=F-\frac{1}{\rho} \operatorname{grad} P+v \Delta^{2} \vec{V} ; \\
& \operatorname{div} \vec{V}=0,
\end{aligned}
$$

но при этом $\mu$ определяется из выражения (8).

Как указывалось выше, практическое значение имеет слоистое течение, известное как течение Куэтта [11]. В случае ньютоновской жидкости решение имеет вид (рисунок)

$$
U=-\frac{1}{2 \mu} \frac{d P}{d x}\left(b^{2}-y^{2}\right)
$$

Для неньютоновской жидкости существенную роль играет зависимость $\mu$ от скорости деформации. В том случае, когда $\mu$ возрастает с увеличением деформации пропорционально градиенту скорости, возникает добавка

$$
U=\frac{2 \sqrt{2}}{3} \frac{d P}{d x} K\left(b^{\frac{3}{2}}-y^{\frac{3}{2}}\right),
$$

где $K$ - коэффициент, зависящий от структуры потока ВУС. 


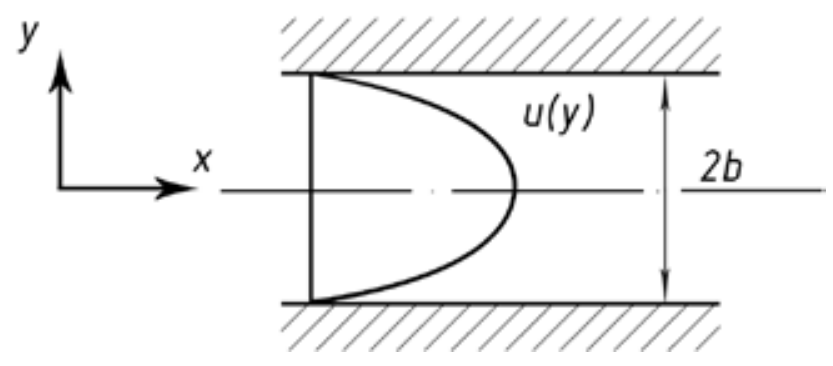

Рис. К расчету течения ньютоновской жидкости

Fig. For calculation of a current of the Newtonian liquid

Сопоставляя полученные результаты с литературными данными [7], для ламинарного потока ВУС нашли удовлетворительное соответствие. Следует отметить, что речь идет о деформации профиля скоростей.

При движении ВУС в тонких каналах, если существует зависимость (6), уравнение (15) должно быть записано в виде

$$
\frac{d P}{d x}=\frac{1}{K \frac{d U}{d y}} \frac{d^{2} U}{d y^{2}} ;
$$

откуда

$$
\frac{d^{2} U}{d y^{2}}=K \frac{d P}{d x} \frac{d U}{d y}
$$

обозначая $K \frac{d P}{d x}=K_{1}$, тогда $\frac{d^{2} U}{d y^{2}}=K_{1} \frac{d U}{d y}$. Если ввести $\frac{d U}{d y}=f$,

то, интегрируя выражение $\frac{d f}{d y}=K_{1} f$, получим

$$
\begin{aligned}
& f=c k e^{k y}, \\
& U=c_{1} k_{1}^{2} e^{k_{1} y}+c_{2} .
\end{aligned}
$$

Используя граничные условия

$$
\left\{\begin{array}{l}
c_{1} k_{1}^{2} e^{k_{1} b}+c_{2}=0 \\
c_{1} k_{1}^{2} e^{-k_{1} b}+c_{2}=0,
\end{array}\right.
$$

находим

$$
c_{1} k^{2} \operatorname{sh} k_{1} b=0
$$

Отсюда очевидно равенство

$$
\begin{aligned}
& \operatorname{sh}_{1} b=0, \\
& k_{1} b=\pi n,(\text { где } n=1,2,3 \ldots)
\end{aligned}
$$


или

$$
K \frac{d P}{d x} b=\pi n
$$

Таким образом, получено уравнение (22), в котором связаны структурный $K$, режимный $\frac{d P}{d x}$ и геометрический $b$ параметры.

Проведенные исследования показали, что при течении ВУС по тонким каналам необходимо учитывать неньютоновский характер ВУС. Установлено, что при движении таких жидкостей нарушается режим течения, что обусловлено взаимным влиянием структурного $K$, режимного $\frac{d P}{d x}$ и геометрического $b$ параметров. Поэтому конструктивные размеры, особенно выходные диаметры отверстий устройств для распыливания ВУС, необходимо подбирать, учитывая полученные соотношения.

Для инженерных расчетов необходимо проводить модельные и натурные эксперименты с конкретными типами форсунок для определения структурного параметра $k$, зависящего от структуры потока ВУС.

Исследование выполнено при финансовой поддержке Российского фонда фундаментальных исследований, Правительства Красноярского края, Красноярского краевого фонда поддержки научной и научно-технической деятельности» в рамках научного проекта № 17-48-240386 p_a.

\section{Список литературы}

[1] Осинцев В.В., Джундубаев А.К., Хидиятов А.М., Козьмин Г.В., Костовецкий С.П. Применение прогрессивных технологий подготовки и сжигания основных энергетических углей Киргизии. Фрунзе: Илим, 1989. 187 с. [Osintsev V.V., Bulakh K.G. Physico-chemical properties of minerals and components of solutions, Leningrad, Nedra, 1989, 187 p. (in Russian)].

[2] Осинцев В.В., Джундубаев А.К., Торопов Е.В., Кузнецов Г.Ф., Сулейменов К.А. Энергоэкологические проблемы сжигания твердого топлива в котельных установках. Челябинск: Издво ЧГТУ, 1995. 162 с. [Osintsev V.V., Dzhundubaev A.K., Toropov E.V., Kuznetsov G.F., Suleimenov K.A. Energy-ecological problems of burning solid fuel in boiler plants. Chelyabinsk: Publishing house ChSTU, 1995. 162 p.].

[3] Кулагин В.А. Методы и средства технологической обработки многокомпонентных сред с использованием эффектов кавитации, дисс. ... д-ра техн. наук. Красноярск: КГТУ, 2004. 379 c. [Kulagin V.A. Methods and means of technological processing of multicomponent media using the effects of cavitation, diss. ... Dr. techn. sciences. Krasnoyarsk: KSTU, 2004. 379 p. (in Russia)].

[4] Баранова М.П. Технология получения нетрадиционных топлив в виде водоугольных суспензий из углей различной степени метаморфизма, дисс. ... д-ра техн. наук. М.: МЭИ, 2014. 275 c. [Baranova M.P. The technology of obtaining non-traditional fuels in the form of water-coal suspensions from coals of various degrees of metamorphism, diss. ... Dr. techn. sciences. Moscow: MEI, 2014. 275 pp. (in Russia)].

[5] Baranova M.P., Kulagin V.A., Taraban'ko V.E. Nature of stabilization of water-coal fuel suspensions. Russian Journal of Applied Chemistry, 2011, 84, (6), 939-944. 
[6] Зайденварг В.Е., Трубецкой К.Н., Мурко В.И., Нехороший И.Х. Производство и использование водоугольного топлива, М.: Из-во Академии горных наук, 2001. 176 с. [Zaydenvarg V. E., Trubetskoy K.N., Murko V.I., Nehoroshi I.H. Production and use of water coal fuel. M., Publishing house of Academy of mountain sciences, 2001.176 p. (in Russia)].

[7] Мурко В.И., Джундубаев А.К., Баранова М.П., Бийбосунов А.И., Кулагин В.А. Гидротранспортные топливно-энергетические комплексы. Российско-кыргызское научнотехническое сотрудничество в области теплоэнергетики. Красноярск: Сиб. федер. ун-т. 2015. 250 c. [Murko V.I., Dzhundubaev A.K., Baranova M.P., Biibosunov A.I., Kulagin V.A. Hydrotransport fuel and energy complexes. Russian-Kyrgyz scientific and technical cooperation in the field of heat power engineering, Krasnoyarsk, Sib. feder. un-t, 2015. 250 p. (in Russia)].

[8] Лойцянский Л.Г Механика жидкости и газа. М.: Наука, 1978. [Loitsyansky L.G., Mechanics of Fluids and Gas. M., Nauka, 1978. (in Russia)].

[9] Ребиндер П.А. Поверхностные явления в дисперсных системах. М.: Наука, 1978. [Rebinder P.A. Surface phenomena in disperse systems. Moscow, Nauka, 1978. (in Russia)].

[10] Бояркин Л.М. Трубопроводный гидротранспорт твердых материалов. М., 1981. [Boyarkin L.M. Pipeline hydrotransport of solid materials. M., 1981. (in Russia)].

[11] Шлихтинг Г. Теория пограничного слоя. М.: Наука, 1974. [Schlichting G. The theory of the boundary layer. Moscow, Nauka, 1974. (in Russia)]. 